\title{
Implementación de circuitos eléctricos para facilitar el aprendizaje de sistemas algebraicos lineales
}

Implementation of electrical circuits to facilitate the learning of linear algebraic systems

Implementação de circuitos elétricos para facilitar a aprendizagem de sistemas algébricos lineares

DOI: http://dx.doi.org/10.23913/ride.v7i14.272

José Genaro González Hernández

Universidad Tecnológica de Altamira, México

jggonzalez@utaltamira.edu.mx

\section{Resumen}

La enseñanza de los sistemas algebraicos lineales, así como sus técnicas de solución, constituyen un eslabón importante en el desarrollo profesional de los alumnos de ingeniería y áreas afines, sin embargo, la asimilación de conceptos se ve afectada por la falta de vinculación entre la teoría y la práctica, de modo que gran parte de los estudiantes muestran con frecuencia poco interés en la explicación de los temas y esto da como resultado un bajo desempeño académico. Este trabajo presenta una propuesta de enseñanza de sistemas algebraicos lineales, en donde se verifican los resultados a través de simulación electrónica e implementación física de circuitos eléctricos para estudiantes de ingeniería.

Palabras clave: enseñanza, aprendizaje, sistemas lineales, circuitos eléctricos. 


\section{Abstract}

The teaching of the linear algebraic systems, as well as its solution techniques, constitute an important link in the professional development of students in engineering and related fields, however, the assimilation of concepts is affected by the lack of connection between theory and practice, so that much of the students often show little interest in the explanation of the issues and this gives as a result a poor academic performance. This paper presents a proposal of teaching of linear algebraic systems, where the results are verified through electronic simulation and physical implementation of electric circuits for engineering students.

Key words: teaching, learning, linear systems, electrical circuits.

\section{Resumo}

O ensino de sistemas algébricos lineares e suas técnicas de solução, são um elo importante no desenvolvimento profissional dos estudantes das áreas de engenharia e afins, no entanto, a assimilação de conceitos é afetada pela falta de ligação entre a teoria e prática, de modo que a maior parte dos alunos muitas vezes mostram pouco interesse em explicar as questões e isso resulta em mau desempenho escolar. Este artigo apresenta um ensinamento proposta de sistemas algébricos lineares, onde os resultados são verificados por meio de simulação e implementação física circuitos eletrônicos para estudantes de engenharia.

Palavras-chave: ensino, aprendizagem, sistemas lineares, circuitos eléctricos.

Fecha Recepción: Junio $2016 \quad$ Fecha Aceptación: Diciembre 2016 


\section{Introducción}

Con el devenir de los años, el proceso de enseñanza aprendizaje ha sufrido grandes cambios, el modelo por competencias ha alcanzado gran aceptación en las últimas décadas, tomando como base el esquema conceptual, procedimental y actitudinal, y es en este sentido, que para lograr un desarrollo holístico en los estudiantes, es fundamental sentar las bases de un conocimiento integral, basado en la interacción de la teoría y la práctica, utilizando las técnicas y métodos apropiados que faciliten la comprensión y asimilación de conceptos (SEP, 2016).

Son diversas las licenciaturas y materias en donde es necesario resolver sistemas algebraicos lineales, desde el balance de sistemas químicos, hasta sistemas de redes eléctricas complejas ECCE (2016). Tan amplia y variada es la cantidad de aplicaciones en donde se establecen estos sistemas, que su enseñanza constituye una parte medular en el desarrollo académico de los estudiantes, que les permitirá asimilar los conceptos para manejarlos más adelante con destreza, y proseguir a la resolución de problemas más complejos.

La Universidad Tecnológica de Altamira (UTA) y el Instituto Tecnológico de Ciudad Madero (ITCM), al ser parte de las instituciones de educación superior tecnológica en México, poseen licenciaturas que incluyen en sus programas de estudio el manejo de los resistores y fuentes de alimentación, tal como se observa en las áreas de Ingeniería Eléctrica y Electrónica (ITCM, 2016), así como en Mecatrónica y Energías Renovables (UTA, 2016); en las cuales los circuitos eléctricos constituyen un aspecto importante, pues estos presentan un escenario ideal para la implementación de sistemas algebraicos lineales (CGUTyP, 2016) (DGEST, 2016).

Por otra parte, es importante la consideración de un apropiado método de enseñanzaaprendizaje, que le permita al alumno no solo mecanizar los procesos de solución, sino dar un significado real a las soluciones.

La comprensión del significado de los sistemas algebraicos lineales y su solución, es un problema no sólo de carácter teórico-conceptual, sino de interrelación con el mundo físico. Los conceptos y formas del lenguaje matemático (con su carácter simbólico y universal), permiten en última instancia, la descripción de diversos fenómenos físicos, completamente reales y tangibles, que son explicados a través de las leyes que rigen el universo. Con base en 
observaciones realizadas a alumnos de diversas licenciaturas del ITCM y la UTA, se encontró que, en general, presentan diversos problemas para la solución de sistemas algebraicos lineales de orden tres o superior, pero aún más, para la extracción de dichos sistemas del mundo real o la interpretación de su significado.

De este modo, se precisó atender esta vinculación entre la teoría y la práctica, para que los estudiantes verificaran los resultados a través de circuitos eléctricos reales, con el apoyo de las herramientas apropiadas.

Tomando todo esto como antecedente, se propuso como objetivo general diseñar un método que permitiera a los alumnos comprender el significado de los sistemas algebraicos lineales, mientras que de forma particular, se analizaron los elementos de configuración para la implementación de un sistema físico real caracterizado por dichos sistemas. También se establecieron los instrumentos de medición apropiados que se aplicaron a los grupos de experimentación y control en el desarrollo de la metodología experimental. Finalmente, se propuso aplicar y evaluar los efectos producidos por el método propuesto, en el desempeño académico de los alumnos al trabajar con la implementación de sistemas algebraicos lineales.

\section{Fundamentos teóricos}

Los métodos de las instituciones educativas que toman como fundamento la caracterización, operación y modelado puramente teórico de diversos sistemas, presentan diferencias radicales a los modelos basados en la práctica; los teóricos que sintetizan su labor en el texto únicamente, tratan de articular las leyes fundamentales de la memoria, lo que a nivel de enseñanza universitaria permite, por lo general, que el alumno sólo asimile la repetición mecánica de conceptos sin sentido para él, ya que el eslabón que los une con la práctica se encuentra desarticulado. Aún en la época actual, se encuentran residuos de estos esquemas en una importante cantidad de elementos diseñados con el propósito de mejorar las destrezas académicas; existe por otro lado, una considerable cantidad de docentes que utilizan su propio conocimiento y apuestan en hacer populares técnicas que tienen el potencial de mejorar la asimilación del conocimiento a través de procesos mentales (Gardner, 2005). Por otra parte, cabe destacar que las inteligencias múltiples, juegan un papel importante en el desarrollo del 
conocimiento, de modo que mientras más sentidos se involucren en el proceso de aprendizaje, éste será más significativo (Gamon, 2008).

Existe una marcada tendencia por parte de profesores de matemáticas universitarios para el manejo de algoritmos complejos y poco didácticos, los cuales se alejan de los ejemplos prácticos y sencillos que despiertan el interés de los estudiantes. Cuando los problemas son puramente teóricos, plantean escenarios cuyo sentido no llega a comprenderse o descifrarse por parte del alumno, sin embargo, la correlación entre las matemáticas teóricas y los fenómenos físicos presentes en la práctica, forman un importante escalón en la asimilación de conceptos, además generan interés en la mayor parte de los estudiantes (Tahan, 2009).

Mucha gente piensa con vehemencia, que las matemáticas son poco interesantes, complicadas, áridas e incluso frías, en cambio, hay personas que las consideran poseedoras de una gran utilidad e interesantes, finalmente es una cuestión de óptica, el punto es que las relaciones matemáticas subsisten en el mundo natural y los seres humanos están inmersos en ellas, lo que constituye una certeza innegable, que puede ser aprovechada por el profesor de matemáticas para invitar, a aquellos que tienen un concepto negativo de esta importante ciencia, un escenario que ofrezca la posibilidad de cambiar su perspectiva al reconocer conscientemente su presencia en todo lugar, verificando de esta manera, su aplicación en el mundo real (De la Peña, 2004).

De acuerdo con Stewart (2012), las matemáticas se encuentran vivas y presentes en el mundo que nos rodea, una realidad que se manifiesta de una forma implacable a través de 17 ecuaciones que cambiaron el mundo, las cuales incluyen desde el Teorema de Pitágoras que está presente en los triángulos rectángulos, hasta la Teoría del Caos que modela el cambio de la población de creaturas vivientes cuando existen límites en los recursos disponibles. Todo esto, pone de manifiesto una realidad que puede ser aprovechada por el docente, para hacer brotar en el alumno el interés por las matemáticas y su relación con la naturaleza.

Por otro lado, el análisis de circuitos eléctricos, es el primer curso basado en cálculos y procedimientos que relaciona directamente la teoría con la práctica, en las carreras de Ingeniería Eléctrica, Electrónica y Mecatrónica. Tales cursos, examinan típicamente elementos básicos, como resistores, capacitores e inductores, los cuales, en el estado estable, representan 
sistemas algebraicos lineales complejos, introduciendo a los estudiantes teoremas, como transformación de fuentes y superposición, análisis de mallas y nodal, entre otros. Con frecuencia, los cursos son complementados con experiencias de laboratorio donde los estudiantes ganan experiencia con el manejo de equipo básico, como fuentes de alimentación de Corriente Directa (CD), multímetros digitales, generadores de funciones y osciloscopios, donde examinan sencillos circuitos eléctricos (Becker, 2014).

Existe amplia y creciente literatura que trata sobre el proceso de enseñanza de tales cursos, y quizá, el tema más común en la mayor parte de esta literatura sea la necesidad de ayudar a los estudiante a desarrollar una comprensión conceptual de los circuitos eléctricos y su vinculación con el modelado matemático de los mismos (Gokhale, 1995). También existe evidencia emergente, de intentos para remover la enseñanza tradicional en esos cursos, en un esfuerzo para promover el desarrollo de la comprensión de conceptos intangibles entre los estudiantes (Yadav, 2011).

En la enseñanza de circuitos eléctricos, hay información que revela que muchos estudiantes están poco menos que inspirados en tales cursos, dado a que el alumno es obligado a aprender diversas leyes y métodos de análisis, así como a memorizar gran cantidad de fórmulas, sin haber realizado un esfuerzo por ganar comprensión conceptual (Lawanto, 2012).

La participación activa en el desarrollo de prácticas de laboratorio, combinadas con apropiadas herramientas computacionales que permitan realizar el modelado y solución de sistemas algebraicos lineales, permite al alumno alcanzar un grado de comprensión más elevado de los conceptos fundamentales, al verificar las soluciones obtenidas tanto manual como computacionalmente, y compararlas con los valores reales indicados en los medidores eléctricos. Es importante la participación activa, ya que existe suficiente evidencia de que el complemento de clases teóricas con estrategias de aprendizaje activas, conducen al estudiante a la retención de conocimiento e incremento del saber, a través de procesos científicos y de descubrimiento, de modo que este tipo de aprendizaje es benéfico y superior, si se le compara con los métodos puramente teóricos tradicionales (Becker, 2014). 


\section{Metodología}

En primer lugar se identificaron las características apropiadas del método de enseñanzaaprendizaje propuesto, con el objetivo de mejorar la comprensión de conceptos y fundamentos en los alumnos. Estos elementos, están formados por un conjunto de mecanismos que llevan inherente la construcción de conocimientos, tomando como base la relación existente entre la teoría y la práctica, todo ello bajo un entorno agradable, en donde los pensamientos y la estimulación mediante el involucramiento de la mayor cantidad de sentidos, sean el punto de partida para la construcción holística del conocimiento (Chamorro, 2005).

Todo esto dio como resultado la implementación de sencillos circuitos resistivos con fuentes de alimentación de voltaje de CD, los cuales son representados a través de sistemas algebraicos lineales. El mecanismo consiste en que el alumno realice manualmente los cálculos para la solución del sistema, utilizando los parámetros reales del circuito, para después, comparar los resultados obtenidos con los arrojados por los instrumentos de medición en la práctica. También se realiza la simulación electrónica mediante algún software especializado de circuitos eléctricos, y además, se resuelve el sistema mediante un programa de matemáticas.

El siguiente paso, fue diseñar un instrumento de medición apropiado, que permitiera obtener resultados que reflejaran el desempeño académico de los alumnos, en cuanto a la comprensión de conceptos, modelado y solución de sistemas lineales.

Dentro de las licenciaturas de ingeniería eléctrica, electrónica y mecatrónica, y durante la impartición de las materias donde se manejan los sistemas algebraicos lineales, se seleccionaron dos grupos de alumnos, uno que recibió las clases de forma tradicional (el de control), y otro que fue tratado mediante el método de enseñanza-aprendizaje propuesto; cada grupo fue de 25 alumnos, los cuales fueron examinados para medir su grado de asimilación de conceptos y resolución de sistemas algebraicos lineales.

Finalmente se aplicaron los instrumentos a los dos grupos, justo después de ver los contenidos en clase, posteriormente se midieron los resultados, lo que constituyó una retención de conocimientos a corto plazo; después de dos meses y sin previo aviso, se aplicó una segunda evaluación y se encontraron otros resultados, que midieron el desempeño académico a mediano 
plazo. En cada examen había dos secciones, una centrada exclusivamente en la asimilación de conceptos, y la otra enfocada al modelado y solución de sistemas algebraicos lineales.

Los elementos de evaluación abordados por los reactivos utilizados dentro de los instrumentos de evaluación se indican en la Tabla 1.

Tabla 1. Elementos evaluados por reactivo

\begin{tabular}{|c|c|}
\hline Reactivo & Elemento evaluado \\
\hline $1-5$ & Asimilación de conceptos \\
\hline $5-10$ & Modelado de sistemas \\
\hline $10-15$ & Obtención de resultados \\
\hline $16-20$ & Interpretación y manejo de resultados \\
\hline
\end{tabular}

\section{Discusión}

Una de las fortalezas del estudio, es que mostró que las relaciones propuestas no sólo permitieron al alumno una mejor comprensión de los sistemas algebraicos lineales, sino que además, despertaron su interés al relacionar la teoría con la realidad operante y lo retaron a encarar otros problemas a través de su habilidad para traducir las ecuaciones a significados físicos, motivando su ingenio y creatividad, y desarrollando de este modo en él, elementos importantes para su futura actividad profesional.

Un aspecto que vale la pena recalcar, es que el estudio se aplicó en estudiantes de dos diferentes instituciones educativas, obteniendo resultados muy similares, aunque se tuvieron limitaciones al no poder extender la investigación a todos los alumnos disponibles, debido a que no todos los profesores que impartían las materias relacionadas con el estudio estuvieron dispuestos a participar.

En cuanto a las áreas de debilidad, es importante considerar que como en todo estudio social, existen características inherentes a cada uno de los alumnos, que no pueden ser controladas por el investigador y que pueden causar cierto sesgo en las mediciones, tales como la condición socioeconómica, conocimientos previos, cultura, motivación y formación sociocultural, por mencionar algunos. También es importante contar con la tecnología informática adecuada, así 
como con los laboratorios, equipos y espacios apropiados para que una investigación como esta pueda realizarse exitosamente.

\section{Conclusiones}

Los resultados fueron satisfactorios, ya que se presentó un incremento importante en cuanto a la asimilación de conceptos y modelado de sistemas por parte de los alumnos del grupo de experimentación. La interacción con los instrumentos de medición en la implementación física de los circuitos eléctricos y el contacto complementario con el software de simulación electrónica y de matemáticas, motivaron el interés de los estudiantes para la solución de los sistemas algebraicos lineales generados en los circuitos resistivos.

En la primera evaluación, el grupo "A" (que fue el grupo de control), obtuvo una calificación aceptable de 82 en cuanto a la asimilación de conceptos, mientras que el segundo grupo obtuvo un 89 en ese mismo rubro, de modo que no hubo una diferencia notoria, sin embargo, los resultados del examen aplicado dos meses después, marcaron una diferencia de 24 puntos entre los promedios de ambos grupos, de modo que el grupo "A" olvidó gran parte de los conceptos, mientras que el " $\mathrm{B}$ " casi mantuvo su rendimiento.

La Tabla 2 muestra un resumen de los resultados de la investigación, en donde la columna "asimilación de conceptos a corto plazo" muestra el resultado de la primera sección del primer examen aplicado. La columna "asimilación de conceptos a mediano plazo" muestra los resultados de la primera sección del segundo examen aplicado dos meses después del primero en ambos grupos.

La columna "modelado y solución de sistemas a corto plazo" establece los resultados obtenidos en la segunda sección del primer examen, mientras que la columna "modelado y solución de sistemas a mediano plazo" revela los resultados de la segunda sección del examen aplicado dos meses después a los dos grupos. La fila A muestra los resultados obtenidos por el grupo de control, mientras que el B indica los resultados obtenidos por el grupo de experimentación.

Dado que los grupos estuvieron formados por alumnos con características similares, los resultados obtenidos en la investigación, implican que el método de enseñanza-aprendizaje propuesto fue exitoso en el desempeño académico de los estudiantes. 
Tabla 2. Promedios de evaluaciones aplicadas por indicador en cada grupo.

\begin{tabular}{|c|c|c|c|c|}
\hline \multirow{2}{*}{ Grupo } & \multicolumn{4}{|c|}{ Indicadores de desempeño académico } \\
\cline { 2 - 5 } & $\begin{array}{c}\text { Asimilación } \\
\text { de conceptos a } \\
\text { corto plazo }\end{array}$ & $\begin{array}{c}\text { Asimilación de } \\
\text { conceptos a } \\
\text { mediano plazo }\end{array}$ & $\begin{array}{c}\text { Modelado y } \\
\text { solución de } \\
\text { sistemas a corto } \\
\text { plazo }\end{array}$ & $\begin{array}{c}\text { Modelado y } \\
\text { solución de } \\
\text { sistemas a } \\
\text { mediano plazo }\end{array}$ \\
\hline A (método tradicional) & 82 & 62 & 61 & 43 \\
\hline B (método propuesto) & 89 & 86 & 85 & 78 \\
\hline
\end{tabular}

La diferencia más grande entre los resultados obtenidos por los grupos de control y experimentación, se dio en el modelado y solución de sistemas a mediano plazo, lo que significa que los estudiantes que no implementaron los circuitos eléctricos, olvidaron en gran medida los conocimientos relacionados con el modelado de circuitos resistivos. Por otro lado, la asimilación de los conceptos a corto plazo, mostró la diferencia más pequeña entre los grupos, la cual fue de siete puntos, lo que indica que de forma inmediata el método propuesto no genera cambios radicales en la comprensión de ideas y cálculos básicos de los sistemas lineales.

En general, los resultados de la investigación arrojaron una mejora de los estudiantes en cada uno de los indicadores propuestos, la implementación y modelado de circuitos eléctricos resistivos, acompañada del uso apropiado de software de simulación electrónica y solución matemática de sistemas, permitieron la construcción holística del conocimiento.

Por razones obvias, no es posible controlar factores inherentes a la naturaleza de los grupos involucrados, tales como los conocimientos previos de cada alumno, sus relaciones interpersonales y entorno social, además de factores diversos y propios de la naturaleza humana que impiden llevar a cabo un verdadero experimento y tener un control absoluto en el tratamiento de las variables. Sin embargo, a pesar de estas circunstancias, los resultados arrojados por el método propuesto son académicamente alentadores. 


\section{Bibliografía}

Becker, J. P., Plumb, C. y Revi, R.A. (2014). Project Circuits in a Basic Electric Circuits Course. IEEE Transactions on Education, vol. 57, no. 2, pp. 75-82.

Chamorro, M.C. (2005). Didáctica de las Matemáticas. España: Editorial Oearson Educación S.A.

CGUTyP, (2016). Coordinación General de Universidades Tecnológicas y Politécnicas. Consultado el 29/05/2016 de: http://www.cgut.sep.gob.mx

De la Peña. J.A. (2004). Álgebra en todas partes. México: Fondo de Cultura Económica.

DGEST (2016). Dirección General de Educación Superior Tecnológica. Consultado el 22 de abril de 2016 de:

http://www.dgit.gob.mx

ECCE (2016). Energy Conversion Congress \& Expo, Montreal, Canadá. Consultado el 18/may/2016 de: http://www.ieee-ecce.org/2016/

Gamon D. y Bragdon A. (2008). ejercicios inteligentes. Ejercicios de acondicionamiento para los seis tipos de inteligencia. México: Grupo Editorial Tomo S.A. de C.V.

Gardner H., (2005). Inteligencias Múltiples. La teoría en la práctica. España: Ediciones Paidós Ibérica: S.A.

Gokhale, A. A., (1995). Collaborative learning enhances critical thinking J. Technology Education, vol. 7, no. 1, pp. 22-30

ITCM, (2016). Consultado el 14/03/2016 de: http://www.itcm.edu.mx

Lawanto, O., (2012). The use of enhanced guided in an electric circuits class: an exploratory study. IEEE Transactions on Education, vol. 55, no. 1, pp. 16-21. 
SEP, (2016). Secretaría de Educación Pública. Consultado el 17/03/2016 de: http://www.sep.gob.mx

Stewart, I., (2012). In Pursuit of the Unknown. USA: Basic Books.

Tahan, M., (2009). Matemática divertida y curiosa. México: Editorial Océano.

UTA, (2016). Universidad Tecnológica de Altamira. Consultado el 18/may/2016 de: http://www.utaltamira.edu.mx/

Yadab, A., Subedi D., Lundeberg, M.A. y Bunting, C.F., (2011). Problem-based learning: Influence on student's learning in an electrical engineering course. J. Eng. Educ., vol. 100 , no. 2, pp. 253-280. 